\title{
Pathway to Care of Psychiatric Services in Gandaki Medical College Teaching Hospital in Western Nepal
}

\author{
Lamichhane $\mathbf{N}^{1}$, Thapa $\mathrm{DK}^{2}$, Timsina $\mathbf{R R}^{3}$, Sharma $\mathbf{R}^{4}$, Vaidya $\mathrm{L}$ and Subedi $\mathrm{A}^{\mathbf{5}}$ \\ 'Dr. Lamichhane N, Associate Professor, Gandaki Medical College, Pokhara \\ ${ }^{2}$ Dr. Thapa DK, Associate Professor, Gandaki Medical College, Pokhara \\ ${ }^{3}$ Dr. Timasina RR, Lecturer, Gandaki Medical College, Pokhara \\ ${ }^{4}$ Dr. Sharma R, Consultant Psychiatrist, Western Regional Hospital, Pokhara \\ ${ }^{5}$ Dr. Vaidya L, Consultant Psychiatrist, Western Regional Hospital, Pokhara \\ ${ }^{5}$ Dr. Subedi A, Lecturer, Manipal Teaching Hospital, Pokhara
}

\begin{abstract}
Key Words
Pathway to care, no prior treatment, psychiatric patients.
\end{abstract}

\section{Correspondence to:}

Dr. Nirmal Lamichhane

Associate Professor and Head

Department of Psychiatry, GMCTH, Pokhara

Email:drnrmlam@hotmail.com

\section{ABSTRACT}

Aims and Objectives: The sequence of contacts with individuals and organizations prompted by the distressed person's efforts, and those of his or her significant others, to seek help is known as the pathways to care. The study of the pathways to care provides the relevant information regarding the individual's health seeking and illness behaviour. A wide range of professionals including psychiatrists, psychologists, other doctors, faith-healers and religious healers cater to the needs of mentally ill patients. This study was carried out to identify the pathway to care of patients up to psychiatric care with no prior psychiatric treatment at GMCTH.

Methodology: It is a cross-sectional, descriptive study conducted in GMCTH, Pokhara from Jan 2015 to Dec 2016. After ethical consideration, all new patients and those newly referred to the psychiatric services and agreed to participate were interviewed until the target 100 participants were recruited. ICD-10 was used for diagnosis. Data were computed and analyzed by using Microsoft excel and SPSS. Parametric \& non parametric statistical technique was applied wherever appropriate.

Results: Total of 100 patients (56 females, 44 males) was included in the study. Mean age of the entire study cohort was 37.17 years (SD 15.45). The mean no. of months to seek first help from a psychiatrist was 53.03 months (SD: 81.70).

Conclusion: All the patients here eventually made treatment contact with psychiatrists. However, delay was pervasive. Delay in treatment has negative effect in the treatment outcome. The knowledge and recognition of psychological disorders by the traditional healers as well as other treatment providers in the community are crucial for early treatment of psychiatric patients. 


\section{BACKGROUND}

Mental and substance use disorders account for enormous burdens worldwide. In developing countries, these disorders are already leading causes of disability and their number is expected to increase. ${ }^{1}$ Pathways to care refer to the sequence of contacts an ailing person makes with services provided by individuals or organisations, prompted by the effort of the distressed persons and those of his or her significant others, in the process of seeking treatment for the ailment. Sociocultural factors and health service variables such as the organisation, availability, and accessibility of health services exert influence on the pathways patients follow in their route to care. ${ }^{2}$ It is by now widely recognised that there is much psychiatric morbidity in primary care medicine. As much as one-quarter to one-third of all general practice patients in some studies suffer from a mental disorder. ${ }^{3}$ Furthermore up to a fifth of all persons in a community may be suffering from some psychiatric illness..$^{4-6}$ Only a small proportion of these present to the psychiatrist. The rest, mainly those with milder illnesses, either remain untreated or receive some form of treatment in the various primary care and general medical care settings. Various reasons have been given for this - the illness may be unrecognized, the stigma attached to a visit to the psychiatrist, or the forbidding distance to the psychiatric clinics. Alternatively cultural explanations for the illness may prevent the patients from seeking western medical treatment. ${ }^{7}$ The aim of the present study was to trace the help-seeking pathway of patients with no prior contact with psychiatric service and to elucidate the role of different services that the patients go through in the process.

\section{MATERIALS AND METHODOLOGY}

It is a cross-sectional, descriptive study conducted in Gandaki Medical College Teaching Hospital, Pokhara from Jan 2015 to Dec 2016. It is a tertiary level, referral health institute in Western Nepal. Taking account of ethical issues, a sample size of 100 was estimated to be sufficient for a meaningful analysis. All new patient or patients newly referred to the psychiatric services and agreed to participate were interviewed until the target 100 participants with no prior psychiatric treatment history were recruited. The data collection was based on the convenient sampling method. Subjects were included in the study, after their informed consent. Those subjects who had been old cases at this facility or had attended any other specialty psychiatric health facility were not included. The clinical information and the interview schedule were used for data collection by interviewing either the patient or the informant or both, as appropriate. In case, it was noticed that patient was not able to give the correct history, the informants were interviewed and efforts were made to verify that information. As the cases were worked up in detail; if at any stage of the study it was found that it was not the first contact with any psychiatric health facility, those subjects were not included in the final data analysis. The diagnosis was finally given, after complete history and examination, on the basis of ICD-10 criteria. ${ }^{8}$ The study protocol was approved by the institutional review committee of Gandaki Medical College Teaching Hospital in Pokhara. Data were computed and analyzed by using Microsoft excel and statistical package for social sciences version 10 (SPSS 10.0). ${ }^{9}$

The basic characteristics of the subjects were presented as a proportion. For categorical variables, interdependence was tested by the chi square test. In some analysis, the groups were clubbed to meet the criteria for the chi square test. For scale variables, an independent sample t-test was used to determine the significance of the difference between the two means. A P value of $<0.05$ was considered statistically significant with $95 \%$ Confidence Interval (CI).

\section{RESULTS}

A total of 136 subjects were enrolled in this study; however, the final analysis was done for 100 subjects (thirty six were excluded, as they were later on found to have attended another psychiatric health facility). Mean age of the entire study cohort was 37.17 years (SD 15.45). The majority of these patients were in the age group of 21 to 50 years (72\%); majority of them were female (62\%), from the rural area (86\%), medium socioeconomic status $(60 \%)$ and literate $(64 \%)$. (Table 1$)$

Most of those were suffering from anxiety spectrum and mood disorders (77\%). Only four (4\%) patients consulted a psychiatrist as the first contact, while $38(38 \%)$ of the cases first consulted traditional healers. 
The mean time patients took to reach the psychiatrist was found to be more when the first helping agencies were traditional healers (67.7 months) or other doctors (58.3 months), as compared to the other agencies. This difference was also statistically significant. The mean number of months to seek first help from a psychiatrist was 53.03 months (SD: 81.70) and equally worrisome was the mean number of months required to seek first help from a psychiatrist even among patients who were seen by other doctors (58. 3 months) while paramedics were better as referral source (25.1 months). (Table 2, 4, 5)

Table 1 Socio-demographic/economic characteristics

\begin{tabular}{|c|c|c|c|}
\hline Item & Category & Frequency & Percentage \\
\hline \multirow{2}{*}{ Sex } & Male & 38 & 38 \\
\hline & Female & 62 & 62 \\
\hline \multirow{6}{*}{ Age in Years } & $<20$ & 12 & 12 \\
\hline & $21-30$ & 24 & 24 \\
\hline & $31-40$ & 27 & 27 \\
\hline & $41-50$ & 21 & 21 \\
\hline & $51-60$ & 10 & 10 \\
\hline & $>61$ & 6 & 6 \\
\hline \multirow{7}{*}{ Education } & Illiterate & 36 & 36 \\
\hline & Literate & 64 & 64 \\
\hline & Primary (I-IV Std) & 21 & 21 \\
\hline & Lower Secondary (V-VIII) & 19 & 19 \\
\hline & Secondary (IX-X) & 9 & 9 \\
\hline & SLC/SEE & 3 & 3 \\
\hline & Graduate $(\geq X+2)$ & 12 & 12 \\
\hline \multirow{3}{*}{ Marital status } & Unmarried & 18 & 18 \\
\hline & Married & 79 & 79 \\
\hline & Separated/Divorced & 3 & 3 \\
\hline \multirow{3}{*}{ Occupation } & Unemployed & 5 & 5 \\
\hline & Partially Employeda & 84 & 84 \\
\hline & Employed & 11 & 11 \\
\hline \multirow{3}{*}{$\begin{array}{l}\text { Socioeconomic } \\
\text { Statusb }\end{array}$} & High & 6 & 6 \\
\hline & Medium & 60 & 60 \\
\hline & Low & 34 & 34 \\
\hline \multirow{2}{*}{ Domicile Settings } & Rural & 86 & 86 \\
\hline & Urban & 14 & 14 \\
\hline
\end{tabular}

Note:

a. Operational definition of partially employed: Partially Employed are those who work but not as fixed job or salaried workers.

b. Operational definition Low, Middle and High Socioeconomic status: Those whose monthly earnings are < 10,000, 10-20000 and > 20000/equivalent Nepalese Rupees.
Table 2 :Distribution of ICD-10 Diagnosis and first pathway of care

\begin{tabular}{llll}
\hline Item & Category & Frequency & Percentage \\
& F20-29 & 2 & 2 \\
& F30-39 & 23 & 23 \\
ICD 10 & F40-48 & 54 & 54 \\
& F60-69 & 2 & 2 \\
& G 43 & 17 & 17 \\
& Deferred & 2 & 2 \\
& Total & 100 & 100 \\
& Traditional Healers & 38 & 38 \\
& Paramedics & 18 & 18 \\
First Contact & Other Doctors & 37 & 37 \\
& Hospital ER & 3 & 3 \\
& Psychiatrists & 4 & 4 \\
& Total & 100 & 100 \\
& Self/Family & 92 & 92 \\
First Contact Suggested By & Friends/Others & 8 & 8 \\
& Total & 100 & 100 \\
\hline
\end{tabular}

Pertaining to the use of psychotropic medications, 50\% of the subjects were given psychotropic medications by other practitioners. Regarding benzodiazepine use alone or in combination, the Fisher's exact test, the twotailed $\mathrm{P}$ value equals 0.0071 . The association between Benzodiazepine uses versus non use is considered to be statistically significant (P value=0.0071). (Table 3)

Table 3: Allopathic Treatment provided

\begin{tabular}{lccc}
\hline Category & Freq & Percent & P-value \\
Benzodiazepine only & 13 & 13 & \\
Benzodiazepine/Antidepressants & 5 & 5 & \\
$\begin{array}{l}\text { Benzodiazepine/Antidepressants/Antipsy- } \\
\text { chotics }\end{array}$ & 3 & 3 & \\
Benzodiazepine/Antidepressants/ Others & 9 & 9 & \\
Benzodiazepine/Others & 10 & 10 & 0.0071 \\
Antidepressants only $\quad$ Stabilizers & 6 & 6 & \\
Antidepressants/Antipsychotics/Mood & 1 & 1 & \\
Antidepressants/Others & 1 & 1 & \\
Antipsychotics only & 2 & 2 & \\
Others only & 29 & 29 & \\
No data & 21 & 21 & \\
\end{tabular}

Table 4: Time to First Contact (in months)

\begin{tabular}{llll}
\hline First Contact & No & Mean & Std. Dev \\
Traditional Healers & 38 & 18.4 & 42.1 \\
Paramedics & 18 & 10.9 & 14.6 \\
Other Doctors & 37 & 17.4 & 41.1 \\
Hospital ER & 3 & 8.7 & 13.3
\end{tabular}


Table 5: Time between First Contact to Psychiatrist (in months)

\begin{tabular}{lccc}
\hline First Contact & No & Mean & Std. Dev \\
Traditional Healers & 38 & 67.7 & 107.4 \\
Paramedics & 18 & 25.1 & 28.6 \\
Other Doctors & 37 & 58.3 & 70.9 \\
Hospital ER & 3 & 34.7 & 53.1 \\
\hline
\end{tabular}

\section{DISCUSSION}

The possible reasons for most of the subjects in this study being 21 to 50 years may be because this is the economically productive age group; therefore, these patients have been brought for the right care. Nevertheless, the presentation of anxiety and mood disorders more in the relatively younger age group ( $<40$ years) has also been reported by authors in the past. ${ }^{10-12}$

The distribution in this study is skewed toward females (62\%), which might be attributed to the prevailing society belief where male member is considered to be stronger than that of a female. Moreover, most of the male members of the country are working abroad. The findings in the present study also support the fact that psychiatric hospital services are utilized more by female patients than by male patients. Other studies have made similar observations. ${ }^{13,14}$

The majority of patients in our study belonged to the middle socio-economic status, with low level of literacy, and was with a rural background. It has been noted that patients belonging to families from the urban, literate, and higher socio-economic status, preferred to take treatment from private practitioners or from distant centers. This could be due to the perceived stigma associated with mental illnesses and with that of psychiatric departments. Predominance of anxiety and mood disorders among these patients may indicate that patients with severe mental illnesses seek treatment from a specialty psychiatric hospital, as there is a common myth that psychiatric hospitals are for mentally ill persons, (patients having psychotic disorders) rather than for patients with any other psychiatric illness. Similar findings have been reported by other authors in India. ${ }^{15}$ In the present study, for psychiatric illness, most cases contacted traditional healers as the primary helping agency. However, subsequently, after not getting any relief, they sought the help of other agencies, such as, paramedics, other doctors, hospital ER, and so on. The psychiatrist was taken as a last resort when all other treatments had failed. In a study in India due to the paucity of facilities, $80 \%$ of the populations have to depend on available treatments consisting of Ayurvedic and Unani systems of medicine, religious treatments consisting of prayers, fasting, and so on, as also various witchcrafts and magical rituals. ${ }^{16}$ The situation is more or less the same in Nepal, and not surprisingly $38 \%$ of the cases in our study contacted traditional healers as the primary helping agency. Another study has noted that a substantial number of patients suffering from severe mental disorders seek other doctors care ${ }^{17}$ Although, the ancient wisdom may have some role in the treatment of mental disorders, ${ }^{18}$ there is a need for generating awareness in the psychiatric patients in Nepal to get professional help. The traditional healers, while dealing with psychiatric patients, often hide their inability to understand and treat these disorders and attribute them to supernatural causes, further enhancing the disbeliefs of these patients. A study on the indigenous healers observed that psychiatric patients used to go through different traditional and faith healers, including indigenous methods of exorcism, before arriving to proper care. ${ }^{19}$ This caused a delay in presentation, which was largely attributable to the stigma associated with such llnesses, ${ }^{20}$ which in turn, led to suffering, and affected the outcome.

In our study, the delay in initiation of proper psychiatric treatment was noted. It was the long path that most of the patients took to reach psychiatrists, and there was a significant delay of up to 396 months, with a mean delay of 53.03 months (4.4 years).

Although it is an established fact that in a majority of psychiatric illnesses, (including schizophrenia, affective disorders) early diagnosis and treatment can significantly improve the outcome and prognosis, Gater et al ${ }^{21}$ have noted that there is a longer delay on pathways involving native healers; while Gureje et $\mathrm{al}^{22,23}$ and Banerjee and Banerjee4 from India, have found that the patients who first consulted traditional healers, tended to arrive at a tertiary psychiatric service much later than those who consulted other caregivers. The results of our study corroborate these findings.

Another important point noted in our study was that the previous patients and their relatives (included in category 'others') were the major referring agency for patients to go 
for psychiatric care followed by allopathic practitioners. These findings may be because of circumstance. In tertiary hospitals other practitioners are major referral source especially with mild to moderate psychiatric problems like anxiety spectrum disorders and mild to moderate mood disorders, as in this study.

Like a study in 1998 which observed that $25 \%$ of the patients took their own initiative to seek help and more than $55.2 \%$ were referred by the spouse or relatives; our study findings was $92 \%$ referrals by self and families. ${ }^{25}$ While another study observed that the medical sector was the most common source of referral.10 In our study, as 57 $\%$ first contacts were paramedics and other doctors, they are the likely source for early contact with psychiatric facilities. Major concern is the mean time taken.

Regarding use of psychotropic medications, $50 \%$ of the subjects were given psychotropic medications by other practitioners. Prescription of benzodiazepine was statistically high with P-value 0.0071. In a hospital based study in Gandaki Medical College, there was a huge variation regarding the duration of benzodiazepines use, ranging from the period of less than of one month to the maximum duration of 192 months or 16 years. ${ }^{25}$ The frequency of benzodiazepine prescription in this study is startling.

The limitation of this study was that it was conducted at a medical college only; study findings may not be reliable and generalization to other centres cannot be made. So, a study from government hospitals, where more representative population attends the OPDs or a multicentre study could have given different results. Secondly, prefixed days are usually attended by the same specialist always; therefore, attendance to such prefixed days may also depend upon the perceived expertise and professional reputation of the expert, among the general population. Third, the patient groups used in this study were heterogenous and the pathway to care may have affected them. As severe mental disorders, which were less represented in this study, are the ones who are likely to reach mental health services as first contact.

\section{CONCLUSION}

The study found that the majority of patients attending the teaching hospital, psychiatric department suffered from anxiety and mood disorders and belonged to the female gender, rural locality, lower socioeconomic class, and was with a low educational status. Traditional healers were the most commonly sought primary helping agency among the study subjects. Pathways involving traditional healers, paramedics and other doctors took a longer time to reach the right psychiatric help. The need for incorporating an efficient and effective referral mechanism, the role of various service providers in the pathway of care, and availability of services should be kept in mind when preparing any mental health program in Nepal.

\section{ANNEXURE}

\section{Definitions}

1. The Psychiatrist in this study was the person who had completed three years of post-graduation studies in Psychiatry from an institute recognized by the Nepal Medical Council.

2. Paramedic was anybody with degrees or certificates recognized by Nepal Health Professional Council.

3. Other doctors were anybody who had at least completed graduation in Medicine and received the degree of MBBS from any medical college recognized by the Nepal Medical Council. However, this person would not have specialized in Psychiatric Medicine.

4. Traditional healers were the people who practiced "dhami", "jhakri", witchcraft, and treated patients by using magico-religious practices. They did not have any medical qualification.

\section{Source of Support: Nil,}

Conflict of Interest: None declared

\section{REFERENCES}

1. Murray CJL, Lopez AD. Mortality by cause for eight regions of the world: Global Burden of Disease Study. Lancet. 1997;349:1269-76.

2. Rogler LH, Cortes DE. Help-seeking pathways: A unifying concept in mental health care. Am J Psychiatr. 1993;150:554-61.

3. World Health Organization. Mental health: New understanding, New hope: World Health Report 2001. 
Geneva: World Health Organization; 2001. p. 09-24.

4. Banerjee T, Banerjee G. Determinants of help-seeking behaviour in cases of epilepsy attending a teaching hospital in India: An indigenous explanatory model. Intl J Social Psychiatry. 1995;41:217-30.

5. Goldman HH. Psychiatric Epidemiqlogy \& Mental Health Research. In: Goldman HH ed. Review of General Psychiatry. Connecticut: Appleton \& Large, 1988; 143-56.

6. Shepherd M. General Practiee, Mental Illness, and the British National Health Service, Am J of Public Health. 1974;64:230-2.

7. Manium T, Ging TS. The prescription of psychotropic medication in general practice - a descriptive study. Med J Malaysia. 1990;45(3):225-30.

8. The ICD-10 Classification of Mental and Behavioural Disorders. Clinical descriptions and diagnostic guidelines. World Health Organization. 2001 pg 6467.

9. Statistical Package for Social Sciences. SPSS version 10.0. USA: SPSS incorporate; 2007. Psychol Med. 1991;21:761-74.

10. Chadda RK, Agarwal V, Singh MC, Raheja D. Help seeking behaviour of psychiatric patients before seeking care at a mental hospital. Int J Soc Psychiatry. 2001;47:71-8.

11. Pradhan SC, Singh MM, Singh RA, Das J, Ram D, Patil $\mathrm{B}$, et al. First care gives of mentally ill patients: $\mathrm{A}$ multicenter study. Indian J Med Sci. 2001;55:203-8.

12. Razali SM, Najib MA. Help-seeking pathways among Malay psychiatric patients. Int J Soc Psychiatr. 2000;46:281-9.

13. Malla AK, Norman RM. Mental hospital and general hospital psychiatric units: A comparison of services within the same geographic area. Psychol and Med. 1983;13:431-9.

14. Worster A, Haines T. Advanced statistics: Understanding Medical Record review (MRR) studies. Acad Emerg Med. 2004;11:187-92.

15. Bhatia MS. Psychiatric morbidity in GHPU adjoining mental hospital. J Mental Health Human behavior.
$2001 ; 6: 114-8$

16. Bagadia VN, Shah LP, Pradhan PV, Gada MT. Treatment of mental disorders in India. Progress in Neuropsychopharmacology. 1979;3:109-18.

17. Naqvi HA, Khan MM. Pathway of psychiatric care in Karachi. J Coll Physicians Surg Pak. 2006;16:438-9.

18. Shamasundar C. Relevance of ancient Indian wisdom to modern mental health - A few examples. Indian J Psychiatry. 2008;50:138-43.

19. Bhana K. Indian indigenous healers. South African Med J. 1986;70:221-3.

20. Loganathan S, Murthy SR. Experiences of stigma and discrimination endured by people suffering from schizophrenia. Indian J Psychiatry. 2008;50:39-46.

21. Gater R, de Almeida e Sousa, Barrientos G, Caraveo J, Chandrashekar CR, Dhadphale M, et al. The pathways to psychiatric care: A cross-cultural study. Psychol Med. 1991 Aug;21(3):761-74.

22. Choo CH. Profile of patients seeking psychiatric treatment from the adult public mental health services in Singapore. Ann Acad Med Singapore. 1997;26:4438.

23. Gureje O, Acha RA, Odejide OA. Pathways to psychiatric care in Ibadan, Nigeria. Trop Geography Med. 1995;47:125-9.

24. Boey KW. Help-seeking pattern of psychiatric outpatients in urban China. Int J Psychiatr Nurs Res. 1998;4:433-43.

25. Thapa DK, Lamichhane N, Subedi S. A Hospital Based Study: Is the Prescription of Benzodiazepine Consistent With the Guidelines? Journal of Universal College of Medical Sciences.2014;2(1):30-4. 